\title{
RM04
}

\section{Integrating Stochastic Seismic Inversion into Reservoir Characterisation Workflows}

\author{
R. Moyen* (Hampson-Russell Software \& Services), R. Porjesz (Hampson- \\ Russell Software \& Services) \& A. Bouziat (Hampson-Russell Software \& \\ Services)
}

\section{SUMMARY}

Seismic inversion is a widely used technique, but it is difficult to quantitatively integrate its results in geological or reservoir models. This is in part due to the difference of scales, and to the difficulty in properly modelling the petro-elastic relationships.

We propose to use stochastic seismic inversion, that computes a large number of fine scale models at a scale closer to the geomodel, to quantitatively estimate the uncertainty associated to the inversion process. We then expose how the results from stochastic inversion can be integrated into geomodels, to compute a seismic-driven facies model that accounts for uncertainties. It is also possible to compute porosity models guided by the wells and the seismic, illustrating the value that seismic can bring even when the well density is high. 
Post-stack and pre-stack seismic inversion techniques are now widely used in the oil and gas industry for reservoir characterization. In recent years, we have also seen an increased focus on uncertainty quantification and finer scale modelling, with a growing use of stochastic inversion. Stochastic approaches generate multiple high frequency realizations of elastic attributes, usually P-wave and Swave impedances, that are all consistent with seismic amplitudes and well data. Stochastic inversion is particularly well suited to geomodelling applications as the high frequency nature of the results is closer to the reservoir model scale, reducing the need for downscaling.

Nevertheless, inversion techniques and specifically stochastic inversion techniques still face the challenge of a truly quantitative integration with geomodels, due to the complexity and nonuniqueness of the link between inverted elastic properties and petrophysical properties. With stochastic inversion techniques, the question arises of how to best exploit all the realizations as it is impractical to interpret each realization individually. Moreover, inversion is generally performed on a support that is different from the reservoir model, creating the need for grid-to-grid resampling techniques, as well as accurate time to depth conversion.

In this presentation, we will show different examples illustrating how to address these problems. Examples of stochastic inversion from different geological settings will show how the inversion can be performed in a fine scale stratigraphic grid at a resolution close to the geomodel, and how the elastic impedances can be transformed into lithology predictions using a stochastic facies classification workflow for uncertainty quantification. This is particularly important when the lithology distribution is a governing reservoir factor, such as in turbidite fields.

A case study on a mature carbonate field also illustrates how petrophysical properties such as porosity can be generated from stochastic inversion results. This leads to a more accurate geomodel, where the fine scale information provided by the wells is complemented by seismically-derived attributes. Even though the well density is high, this result will be shown to be more accurate than a geomodel based only on the well information. It also illustrates the need for a high-resolution time to depth conversion to properly calibrate the inversion results to the well data. 